\title{
Autobiographical memory in two older adults over a twenty-year retention interval
}

\author{
LINDA LHOST CATAL \\ University of Detroit Mercy, Detroit, Michigan \\ and \\ JOSEPH M. FITZGERALD \\ Wayne State University, Detroit, Michigan
}

\begin{abstract}
This article reports on a study of autobiographical memory in two older adults, wife and husband, over a retention interval of 20 years. The female participant kept a 20 -year log of daily events. A sampling of events that varied in distinctiveness, from unique, one-of-a-kind events to routine, almost daily events, was used to examine cued recall using an incremental scoring system. Each event recalled was also rated on phenomenological scales of remembering versus knowing, rehearsal, and importance. The strongest effect on recall was the order of the cues, with an initial what cue, containing the content of the event, proving superior to cues containing who or where elements. When was the most effective second cue. The results demonstrate regularity in retention over time and highlighted the utility of this approach for understanding the factors that influence autobiographical memory performance.
\end{abstract}

Autobiographical memory is defined here as recall for personal experience, memory for naturally occurring, "real-world" events (Linton, 1978). The term autobiographical suggests memories that have direct reference to the self (Conway, 1992; Fitzgerald, 1999) and that are stored without benefit of conscious memory activity (Fitzgerald, 1986). Early long-term memory research studied recall for facts and universally experienced events, such as persons in the news and television shows (Squire \& Slater, 1982), U.S. presidents (Roediger \& Crowder, 1982), or a foreign language (Bahrick, 1984). These studies provided useful information about recall of commonly experienced events, but provided limited insight into memory and recall for everyday events in an individual's personal life.

Research on memory for personal events has relied on the cue word approach (Crovitz \& Schiffman, 1974; Fitzgerald \& Lawrence, 1984; Galton, 1879, 1883; Robinson, 1986; Rubin, Wetzler, \& Nebes, 1986), diary studies (Barclay \& Wellman, 1986; Brewer, 1988; Conway, Collins, Gathercole, \& Anderson, 1996; Larsen, 1988; Linton, 1975, 1978; Thompson, 1982; Thompson, Skrowronski, Larsen, \& Betz, 1996; Wagenaar, 1986, 1988; White, 1982), studies of involuntary memories (Bernstein, 1996; Salaman, 1970), and "flashbulb" memory studies (Con-

\footnotetext{
The authors wish to express their appreciation to the participants in this research, who gave so generously of their time and effort in completing this study. We also thank Patricia Siple, David Rubin, and several reviewers for their valuable comments. Correspondence should be addressed to J. M. Fitzgerald, Psychology Department, Wayne State University, Detroit, MI 48202 (e-mail: aa1670@wayne.edu or lhost@ misd.k12.mi.us).
}

way, 1995; Rubin \& Kozin, 1984). Of these, cue word and diary studies are particularly relevant to the present study.

Cue word results have demonstrated several reliable patterns in memory distribution over the life span, including a retention function, in which memory strength declines as a function of time (Crovitz \& Schiffman, 1974; Rubin et al., 1986); an overproduction by middle-aged and older adults of memories from early adulthood called the "reminiscence bump" or "the bump" (Fitzgerald, 1988, 1996; Fitzgerald \& Lawrence, 1984; Rubin et al., 1986); and "childhood amnesia," reflecting a paucity of memories produced prior to age 5 to 7 (Cohen \& Faulkner, 1988; Wetzler \& Sweeney, 1986). With respect to older adults, studies have shown them to be as productive as younger adults in recalling memories with the cue word approach (Fitzgerald \& Lawrence, 1984) and also in their ability to access recent memories (Fitzgerald, 1996).

As rich as cue word data have been, the cue word method has some inherent limitations, primarily due to an inability to verify the accuracy of the memories obtained. Diary studies have better potential for examination of autobiographical memory because keeping a daily log provides precise dating and a verifiable record of episodic events (Linton, 1978; Thompson, 1982; Wagenaar, 1986). Participants keep a daily record, usually of the most memorable event of the day, and the record is used to generate items for later memory testing.

This article reports the results of a diary study of a married couple in their 70s at the time of data collection. They were related to L.L.C., who was aware of the daily $\log$. The wife had kept a diary for 20 years as a means of providing her family with a record of events ranging from important and unique events (weddings, funerals, 
and life events) to the mundane and trivial (sitting on the patio). The diary was not a verbal narrative in the conventional sense, but rather was a log of daily events, similar to the memory records kept in autobiographicalmemory studies by Linton (1978) and Wagenaar (1988). The husband had not logged events, thereby permitting us to contrast recall of a recorder and a nonrecorder.

Long-term diary studies of more than a year, such as ours, are rare because participants do not easily keep daily $\log$ s for extended periods of time. Consequently, the most thorough diary studies thus far have typically been single-participant designs, using middle-aged psychologists as examiner-participants. To date, none of the long-term diary studies have used diarists over the age of 50 , making our nearly 80 -year-old participants unique.

When psychologists study their own memory, rehearsal effects are suspected, and the generalizability of results is open to question (Conway et al., 1996). Concerns about recorder effects arise because it is the diarist-participant who screens and selects events of the day, presumably choosing the most memorable events, and because the act of recording itself is thought to reinforce the recorder's memory.

Thompson (1982) found no difference in recall between diarists and their nonrecorder roommates. Thompson et al. (1996), however, reported modest recorder effects when recorders selected events on the basis of self-relevance rather than mutual participation. It is rare to obtain a long-term diary of events from an inexperienced participant who is naive to the study at the time of event recording, such as in the present study. Although record keeping may be less systematic with such a naive participant, there are also possible advantages, because events are not deliberately screened for memorability. There is less recorder bias because the participant is naive to the experimental design, including how the diary might one day be used or how memory might one day be tested. In the present study, the recorder selected all the events, and even though both individuals experienced many of the events together, it was anticipated that more of the events she selected would have personal relevance and that she would perform at a higher level.

With regard to event selection, most previous diary research has targeted memorable events (Linton, 1978; Thompson, 1982; Wagenaar, 1986), because less distinctive events were easily forgotten or became indistinguishable (Linton, 1975). Overall, there has been limited deliberate memory testing for routine experience, despite the fact that most of life is composed of these nondistinctive events. White (1982) and Brewer (1988), however, deliberately solicited both memorable and mundane events. When a diarist spontaneously keeps a daily $\log$ of multiple events, such as in the present study, there is a large corpus of both mundane and memorable events. We purposefully included a full range of events, unique to routine, with the intent of accurately characterizing recall of personal events.
We quantified the unique-routine continuum by rating each event with regard to how frequently the elements in the event appeared in the log, and we conceptualized the variable as "distinctiveness." Thus, distinctiveness was defined in the context of what was recorded in the log, and not necessarily what took place in the participant's life. Time, cue order, and distinctiveness were examined as contributors to memory performance, along with self-ratings of event rehearsal, event importance, and know-remember, which represented participants' rating of the phenomenological experience of recall, specifically the extent to which recall represented a "reliving" or "reexperiencing" of the original event (Wheeler, Stuss, \& Tulving, 1997).

Using older participants, a longer retention interval, and a full range of events (unique to routine), we hoped to expand on Wagenaar's (1986) work on cue efficiency, Brewer's (1988) work with phenomenological factors in recall, and White (1982) and Brewer's comparison of mundane and memorable events. We hypothesized that the order in which we presented cue information would affect participants' recall. On the basis of Wagenaar (1986), we hypothesized that initial what cues would be the most powerful, with who and where cues showing moderate efficiency, and when being the least effective. By virtue of their closer match with the original encoded material, we knew what cues to be inherently more specific and distinctive and more likely, therefore, to cue the correct event (Tulving, 1983).

Linton (1975) found "one-of-a-kind" events that held significance for her to be more memorable, and so we hypothesized that our 2 participants would remember distinctive events better than routine events. Event importance and event rehearsal (talking or thinking about an event) were expected to enhance memory. An important issue is how the various influences on memory performance interact. In a short-term diary study, Conway et al. (1996) presented a comprehensive evaluation of the predictors of autobiographical memory performance by examining recognition memory for actual events and foils. They reported that recognition of actual events was best when the stimulus created both a sense of familiarity and a sense of recollection, whereas the correct rejection of false memories relied more on a lack of familiarity. Although the variables included in their study and ours overlap, specifically importance and distinctiveness, the studies differ in that we examined recall rather than recognition. In addition, our study spanned 20 years, and our participants performed no ratings at the time of the event.

Figure 1 presents the integrative model of memory performance tested here. In this model, importance and distinctiveness are seen as having direct effects on memory as well as indirect effects moderated by rehearsal. Thus, an important event may be more likely to be recalled both because it related to the completion or frustration of some enduring life goal (Conway et al., 1996) and because such events are more likely to be rehearsed. 


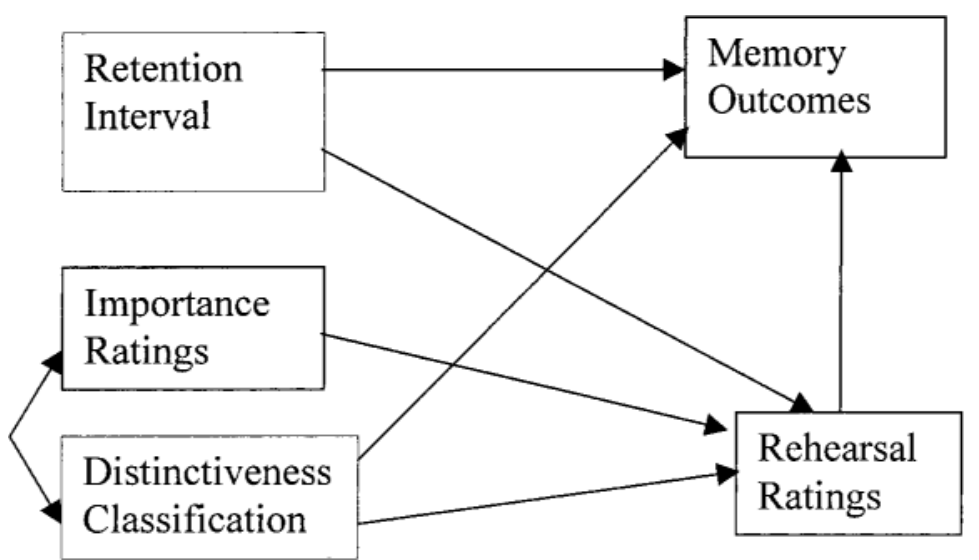

Figure 1. Model of relations among study variables. Memory outcome refers to performance score and ratings on the Know-Remember Scale.

Although retention interval is conceived of as having direct impact on performance, it is probable that the passage of time allows for more rehearsals, and more rehearsal thus moderates the impact of time on memory.

\section{METHOD}

\section{Participants}

Participants were wife and husband of almost 60 years, both college graduates. They were 78 (female) and 79 (male) years old at the time of testing and were healthy, active, older adults. Unlike in previous studies, pretesting of intelligence (WAIS-R), memory (Wechsler Memory Scale-Revised), and personality (MMPI) was undertaken to ensure participants were in the normal range. Across all measures, participants were average or above average, which allowed us to interpret our results as relevant to what should be considered typical for their age group.

\section{Stimuli}

The female participant had kept a daily log of events for a 20-year period, spanning from the late 1970s to the time of testing. As noted above, this was not a narrative diary discussing personal experiences but rather a factual record of daily events that evolved from a routine appointment calendar. Information was entered in the 1-in. squares of a yearly pocket calendar. A typical entry might read "lunch with Ruth at Palm Court." There were varying numbers of events recorded for a given day, anywhere from 2 to 5 . In all there were over 18,000 events recorded, but not all events were usable for testing.

An event, for purposes of testing, was defined as an experience in the log that included all four distinct variables of who, what, where, and when. Events were eliminated if they failed to meet these criteria. For example, there were events in which the elements what and where were somewhat indistinguishable ("went over for dinner"), or the element of who was too vague ("lunch with the gals"). Eliminating events that did not have four distinct elements reduced the total pool of events to between 50 and 60 very routine events per month and 10 to 15 moderately to very distinctive events per month. Where a piece of information could be verified by outside corroboration, the information was included, even though it might not have been specifically stated in the log. For example, "moved into our new house" did not mention the city, but the location was a matter of common knowledge. However, if an event element was entered in the log, it was not modified, nor was additional or more specific information presumed. So, for example, if an event was recorded as occurring in the participants' home, the exact location, kitchen or living room, was not assumed, even if the location was very likely.

Events were conceived as being on a continuum, from unique (one-time event) to routine (almost daily). This continuum is referred to here as distinctiveness. A 4-point scale was used to rate events based on the frequency of the event's occurrence in the log and the number of elements that the event shared with other events. Highly distinctive events were judged to have the four distinct, rarely duplicated elements of who, what, where, and when (e.g., "saw Ken Venturi at breakfast while at Legends of Golf, Austin, Texas") and were rated "4." Very routine events had all three of the exact same elements of who, what, and where and differed only with regard to when (e.g., "did laundry") and were rated "1." With regard to frequency of events in the log, some routine events occurred anywhere from 5 to 25 times per month, some almost daily (e.g., walking). Other events occurred on or around the same day and month, several years in a row (e.g., birthday dinners). Distinctiveness was a summary judgment of each event by L.L.C., assigned following a review of the $\log$ and prior to testing. Ratings were independent of the participants' performance. A test of interrater reliability was conducted on a sample of 60 items, which were rated by L.L.C. and another individual familiar with the log. The result was an intraclass correlation coefficient $(r=.857, p<.01)$. This estimate of reliability was based on a generalizability model in which two initial raters score a subset of items, but only one rates all of the remaining items (Crocker \& Algina, 1986).

Within each 4-month block of time, eight events were randomly selected, with the intention of sampling equally from all four levels of distinctiveness. Totally distinctive events, however, were underrepresented in the log. To compensate, when a higher rating (e.g., an event rated " 4 ") was unavailable, we substituted two events of the next lower rating (e.g., two events rated "3") to maximize average level of distinctiveness for that time period. This was possible for most years except in the earliest part of the log, when record keeping was first beginning. Table 1 provides the frequency distribution of distinctiveness ratings.

The item pool included events for which both participants were originally present, as well as events during which participants were without their spouse (e.g., "I saw Dorothy Belle at bridge luncheon; Harv played golf at River Bend with Jack"). Since she kept the log, the female participant had entered more individualized events for herself than for her spouse. Nevertheless, the individualized event 
Table 1

Distribution of Distinctiveness Ratings

\begin{tabular}{|c|c|c|c|c|c|c|c|c|}
\hline \multirow[b]{3}{*}{ Initial Cue } & \multicolumn{8}{|c|}{ Distinctiveness Rating } \\
\hline & \multicolumn{2}{|c|}{1} & \multicolumn{2}{|c|}{2} & \multicolumn{2}{|c|}{3} & \multicolumn{2}{|c|}{4} \\
\hline & No. & Prop. & No. & Prop. & No. & Prop. & No. & Prop \\
\hline \multicolumn{9}{|c|}{ Female Participants } \\
\hline What & 36 & .18 & 105 & .53 & 49 & .25 & 5 & .05 \\
\hline Who & 42 & .21 & 95 & .47 & 57 & .28 & 7 & .04 \\
\hline Where & 48 & .23 & 112 & .55 & 38 & .19 & 5 & .03 \\
\hline Total & 126 & .21 & 312 & .52 & 144 & .24 & 21 & .03 \\
\hline \multicolumn{9}{|c|}{ Male Participants } \\
\hline What & 31 & .18 & 91 & .52 & 42 & .24 & 11 & .06 \\
\hline Who & 33 & .19 & 92 & .53 & 45 & .26 & 4 & .02 \\
\hline Where & 44 & .25 & 95 & .543 & 31 & .18 & 5 & .03 \\
\hline Total & 108 & .21 & 278 & .53 & 118 & .23 & 20 & .04 \\
\hline
\end{tabular}

pool for the husband was sufficient to eliminate the methodological issue of his events always including his wife among the who. Thus, each participant was tested on a large pool of "shared events" in which they were coparticipants and a smaller pool of events for which the spouse was absent. The two item pools were matched, as closely as possible, with regard to date and distinctiveness.

We intended to select 480 events ( 24 events $\times 20$ years $)$. As noted above, however, items were added to maximize average distinctiveness for each period. This increased the pool of events to 609 for the female and 528 for the male. The number of events per year actually ranged from 22 to 37, with the lowest number occurring during the earliest part of the log, when record keeping was initiated. Five items were eventually deleted due to ambiguous wording, yielding a final item pool of 604 for the female participant and 524 for the male participant (only 4 of the 5 deleted items pertained to the male). For each item that participants remembered, phenomenological data were collected, including measures of event importance, rehearsal, and remember-know, indicating whether the event was actually remembered or just factually known to have occurred (rating scales discussed later).

Testing was conducted over 12 sessions, each 3 to 4 hours in length. Each session was divided into two work blocks, one in the morning and one in the afternoon, following lunch. Written directions were reviewed at the start of each morning session. Participants were encouraged to take small breaks, as needed, to eliminate any confounding effects of fatigue or emotion. The examiner was available throughout testing for questions. Participants reported difficulty differentiating between barely knowing an event to have occurred and having no memory of it at all. Both participants experienced emotional reactions to items that surrounded the death of their mothers, and both took a brief break.

\section{Test Materials}

Retention was tested using Wagenaar's (1986) cued-recall method. A 5-page test booklet was constructed for each event. Each booklet consisted of 4 pages to test memory for the event and 1 page to assess phenomenological data. For each event, four retrieval cues were identified: who, what, where, and when. For events in which the participant was alone, the who was stipulated with the participant's name followed by a hyphen and the word alone (Name-alone). When who included another person on the phone, rather than physically present, this was stipulated (Helen-phone). If the criteria were met, phone events were included in the study. Figure 2 presents samples of the first 4 pages.

Cues were presented to participants in random order, cumulatively, adding one element per page, until all four elements appeared together on the 4th page. Given research to suggest that when was an ineffective cue by itself (Wagenaar, 1986, 1988), when was never given as the first cue. Therefore, the initial cue order varied between the other three cues, yielding 18 different orders.

\section{Scoring}

Autobiographical memory was measured incrementally, by calculating a performance score. Performance scores were calculated by adding the number of correct elements that participants remembered, per event. Possible scores ranged from 0 to 6 and represented an aggregate total of event information recalled accurately over the 3 pages of the response booklet. For example, the 1st page presented the first cue alone (who, what, or where) with three blanks. The task was to recall from memory the three missing aspects of the event ( 3 points possible). Page 2 provided the first cue and a second cue, along with two blanks ( 2 possible points). The 3 rd page listed three cues and one blank (1 point possible). Figure 2 illustrates the testing process.

\section{Rating Variables}

The 4th page contained all four cues (who, what, where, when), and participants were asked whether or not they remembered the event at all. For those events recalled, phenomenological data were collected on page 5 . This consisted of subjective ratings on four 7-point scales: rehearsal-talk, rehearsal-think (measuring frequency of rehearsal since the event took place), importance (measuring the importance or signif icance of the event to the participant), and remember-know (measuring the degree to which participants reexperienced the event at recall). We used the Know-Remember Scale (Hyman, Gilstrap, Decker, \& Wilkinson, 1998). On this scale, higher scores (7) are toward the remember end of the continuum. This scale included a brief statement that defined each end point of the continuum. Knowing (1) was defined as recognizing the event to have occurred but without any recollection or clear memory of the event. Remembering (7) was defined as reexperiencing or having a conscious recollection of some aspect of the event. This might be a visual, auditory, or tactile association, an image of the original event, or reliving an emotion or thought. This continuum approach expands upon laboratory studies that used dichotomous judgments (Rajaram, 1993).

\section{Debriefing}

The female participant described her criteria for entering and censoring events in the log. Although some topics were not included, she reported not deliberately censoring any information and explained she had not anticipated anyone ever reading the logs. Her 
PAGE 1

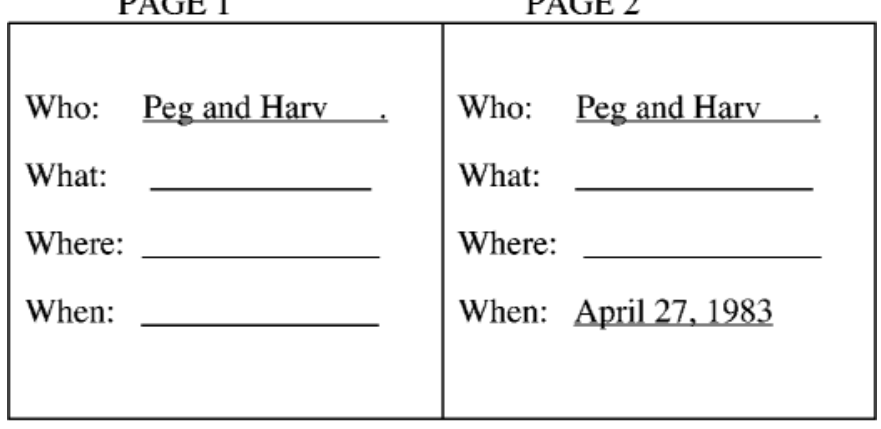

PAGE 3

PAGE 4

\begin{tabular}{|c|c|c|c|}
\hline Who: & Peg and Harv & Who: & Peg and Harv \\
\hline What: & $\begin{array}{l}\text { Legends of Golf } \\
\text { Tournament }\end{array}$ & What: & $\begin{array}{l}\text { Legends of Golf } \\
\text { Tournament }\end{array}$ \\
\hline Where: & & Where: & $\begin{array}{l}\text { Onion Creek } \\
\text { Austin. Texas }\end{array}$ \\
\hline When: & April 27, 1983 & When: & April 27, 1983 \\
\hline
\end{tabular}

Figure 2. Sample response form. The 4 pages on which participants attempted to recall cued information.

intention was to list the events of the day, and given the 1-in. squares in the calendar, she reported brevity as being a result of limited space, not censorship.

\section{RESULTS}

Separate data analyses were conducted for each participant. Analyses included (1) the distribution of performance scores, representing memory strength; (2) the effect of cue order, event distinctiveness, and time on memory; (3) the correspondence of the time data to existing models of long-term retention; (4) phenomenological reports of rehearsal and importance; (5) the correlates of remember-know ratings, and (6) dating accuracy. We note that the units of analysis here are events rather than individuals; that is, the attempted recall of each event is regarded as having been sampled from the population of events in the diary.

\section{Performance Scores}

The distribution of performance scores for both participants is shown in Figure 3. The highest frequency of scores was at the 1-point range, indicating participants were able to recall at least one element for a substantial number of events, $81.5 \%$ for the female and $77 \%$ for the male. Over twice as many items were in the lower point range (1-3 points) compared with the upper range (4-6 points). Frequency distribution of scores showed that multiple cues were required to access most memories. Judging by the low number of 5- and 6-point scores, remembering all or a majority of elements, with only one cue, was quite difficult. On the other hand, both participants were able to provide partial information for a substantial number of events. Mean performance scores were 2.42 (female) and 2.03 (male), which represented a recall rate of $40 \%$ (female) and $34 \%$ (male).

In the first set of analyses, performance score (1-6) was the dependent variable, and event age and cue order were independent variables. The original 18 cue orders were collapsed into three groupings according to the initial cue (who, what, and where). Distinctiveness was entered as a covariate rather than a design variable because the number of events varied within cells defined by time and cue order. As seen in Table 1, there were very few highly distinctive events. The four levels of distinctiveness were not evenly distributed across time. In fact, there were no representatives of the highest distinctiveness level for some time periods. Rather than create a design with empty cells, we opted for analysis of covariance (ANCOVA).

ANCOVA of performance scores revealed a main effect of cue order (who, what, where) for both participants: female $\left[F(2,573)=64.62, p<.001, \eta^{2}=.184\right.$; male, $\left.F(2,492)=28.12, p<.001, \eta^{2}=.103\right]$. There was no effect of retention interval, which was measured in terms of the number of months between the date of the event and the date of testing $(F<1)$. There was a weak interaction 


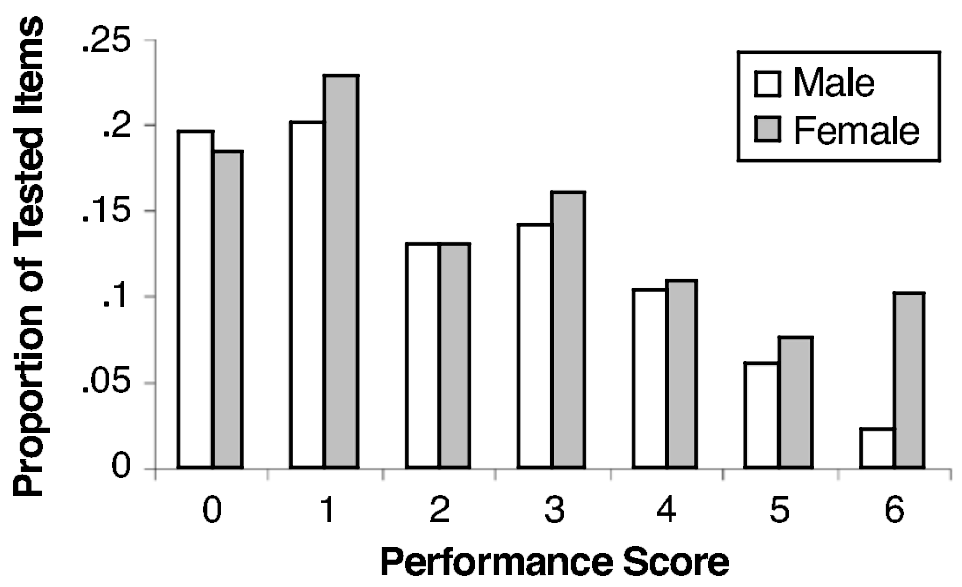

Figure 3. Distribution of performance scores. One point was given for a correct response to each of the blanks in the response booklet with a maximum of 6 points for each event tested.

between cue order and retention interval for the female participant $\left[F(18,573)=1.7, p<.05, \eta^{2}=.05\right]$, but not for the male $(F<1)$. There was a significant effect of distinctiveness for both participants: female $[F(1,573)=$ $49.15, p<.001, \eta^{2}=.079$; male, $F(1,492)=15.76, p<$ $\left..001, \eta^{2}=.031\right]$. These analyses suggest that the cueorder and distinctiveness variables heavily influenced performance, making it difficult to see any effects of retention interval when all orders are collapsed together. This issue will be examined shortly. First, however, we examine the cue-order effects in more detail.

Post hoc analysis using the Scheffé test indicated that initial what cues were significantly more successful as memory cues than either who or where cues. This was true for both participants $(p<.001)$. There was no significant difference between initial who and where cues for either participant $(p>.10)$. The means are presented in Table 2 .

To further understand the cue effects, we tested the hypothesis that the what-when combination would be the most efficient order. Our focus on the what-when combination was intended to replicate Wagenaar (1988). In the present set of events, however, the who and where elements turned out to be less unique and less efficient as retrieval cues. Analysis of first-second cue combinations was conducted by pairing each of the three initial cues (who, what, where) with each possible second cue (e.g., who-what, who-where, who-when). Analysis of

Table 2

Mean Performance Scores for Cue Combinations

\begin{tabular}{lcc}
\hline Cue Order & $\begin{array}{c}\text { Female } \\
\text { Participants }\end{array}$ & $\begin{array}{c}\text { Male } \\
\text { Participants }\end{array}$ \\
\hline What first & 3.65 & 2.86 \\
Who first & 1.87 & 1.69 \\
Where first & 1.76 & 1.55 \\
What - who & 3.57 & 2.81 \\
What when & 4.36 & 3.40 \\
What - where & 3.19 & 2.47 \\
\hline
\end{tabular}

variance (ANOVA) detected at least one cue-order combination to be significantly different from the others. We followed up with a Scheffé post hoc test to determine which orders differed significantly. For both the female and male participant, when the initial cue was either who or where, there was no significant effect of a second cue on memory performance scores $(p>.15)$. However, for events initiated with what cues, there was a significant improvement in memory retrieval when the second cue was when, as opposed to either who (when vs. who $p<$ .05 , both participants) or where (when vs. where, $p<$ .001 , both participants). Results of this analysis supported the prediction that the what-when order would be the most effective in memory retrieval. Means for cue combinations are reported in the lower half of Table 2.

\section{Retention Interval and Performance}

The effect of time on performance scores was difficult to ascertain in the initial analysis of covariance, in part because performance was uniformly low for initial cue orders of who and where. In addition, there was considerable variability among the age intervals in terms of the distinctiveness of the events from the various time intervals. Thus, if a distant interval had many distinct events, performance might be stronger than expected; similarly, if a relatively recent interval had few distinct events, performance might be lower than expected.

In light of the variability in the distinctiveness across retention intervals, performance scores were analyzed using aggregated data. Mean performance scores were calculated for 2-year time blocks and for each of the three initial cues (who, what, and where), as well as for the total data set. Although this served to reduce noise in the data, retention functions for who events and where events continued to show considerable inconsistency, with no systematic linear or curvilinear effect of retention interval on performance scores. Data for what-cued events, however, were orderly and followed the systematic decline in 




Figure 4. Performance scores and retention interval only for those events initially prompted by what cues.

memory observed in previous studies. Performance data for what-cued events are displayed in Figure 4.

Previous studies (Rubin \& Wenzel, 1996) suggest that the relation between time and memory is best fit by a variant of the power model $\{\log$ (memory strength) $=$ $f[\log ($ time $)]\}$, but that a variety of other linear and curvilinear models have fit other data sets. We employed regression analysis to evaluate the power model and the simple log model, as well as several more complex models evaluated by Rubin and Wenzel. For the present data, differences among nonlinear models for both participants were minor (less than $1 \%$ of the variance), so we report only the power model results here. For the events prompted with initial what cues, the linear model accounted for $56 \%(p<.05)$ and $76 \%(p<.05)$ of the variance for the female and male participants, respectively. For the power model, a stronger inverse relation between time and memory strength was found for both the female $\left[F(1,9)=18.16, r^{2}=.69, p<.003\right]$ and the male $\left[F(1,9)=92.75, r^{2}=.92, p<.001\right]$. Although the degree of fit was more modest than that found in studies based on group data, the beta values are similar but shallower than those reported for other studies with longterm retention data (Rubin \& Wenzel, 1996). Those data, however, were for specific information such as the names of high school classmates rather than details of personal events (Bahrick, Bahrick, \& Wittlinger, 1975).

\section{Know-Remember Scale and Age}

Parallel to the analyses of the aggregated performance data, regression analyses were performed on the KnowRemember Scale data to assess the fit of those data to a power function, as well as several alternative curvilinear models, as for memory performance. The fits were equivalent. Figure 5 presents the raw data and the best-fit lines. Analyzing the distribution of mean ratings for the Know-Remember Scale across 2-year time blocks in the power model, the effect of time was significant for both participants $\left[F(1,9)=20.42, r^{2}=.72, p<.002\right.$, for the female $]$ and $\left[F(1,9)=6.97, r^{2}=.39, p<.05\right.$, for the male] In both cases, these curvilinear models were superior to the linear models $\left(r^{2}=.48, p<.025\right.$, and $r^{2}=.24, p>.15$, for the female and male participants, respectively). For the female participant, the slope of the Know-Remember Scale showed a decline $(\beta=-.8476)$ in the degree to which the participants reported reexperiencing the events across the 20 years of the $\log$, with the exception of a spike in ratings for remote events at years $14-15$. The slope for the male participant showed an overall less consistent and more gradual decline. He also had unexpected increases in ratings for the intervals $12-13$ and 16-17 years.

\section{Dating Accuracy}

To evaluate dating accuracy, we assigned each "when" response a value between 0 and $4(0=$ no correct dating information; $1=1$ year off; $2=$ correct year; $3=$ cor rect year and month; $4=$ correct year, month, and date). The distribution of this variable was somewhat skewed for both participants, with a large number of 0 s and very few $4 \mathrm{~s}$.

For each participant a series of bivariate analyses were computed between dating accuracy, retention interval, distinctiveness, importance, and rehearsal. For the female participant, accuracy correlated significantly but weakly $(r=-.06, p<.01)$ with the retention interval; that is, the more distant the memory, the less accurate the 


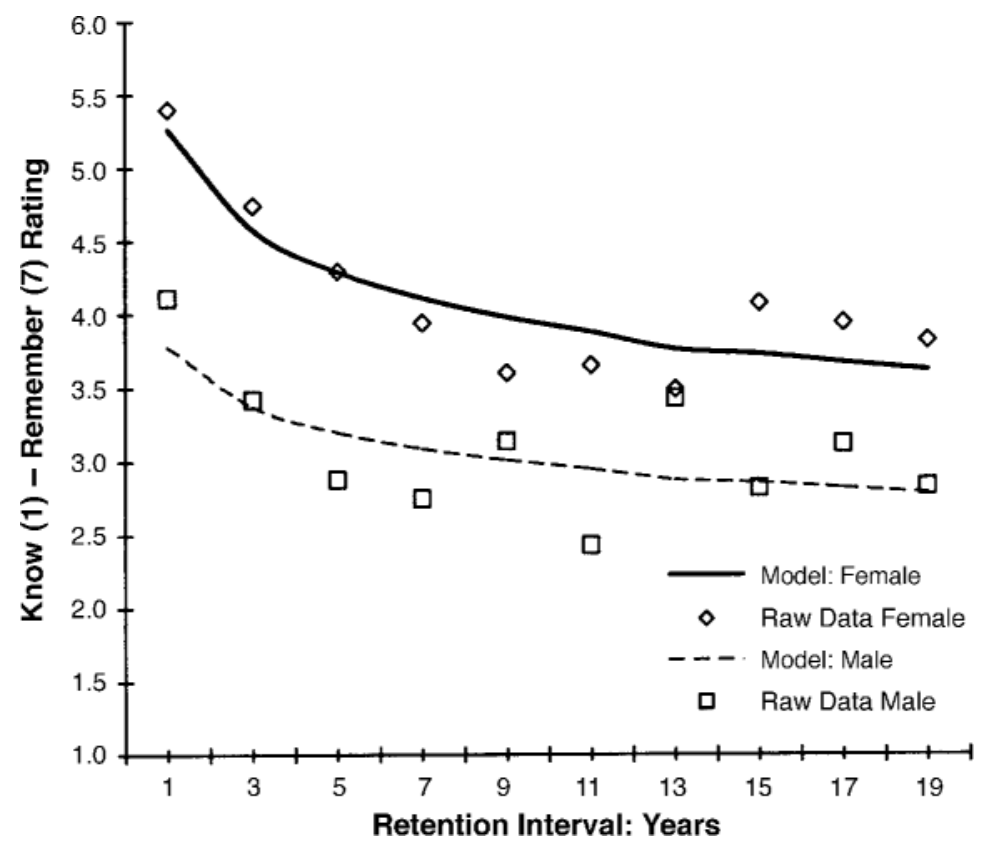

Figure 5. Know-Remember Scale scores and retention interval for all items tested.

date given. Accuracy correlated positively with distinctiveness $(r=.23, p<.001)$, importance $(r=.42, p<$ $.001)$, and rehearsal $(r=.29, p<.001)$. A regression analysis with these four variables (retention interval, distinctiveness, importance, and rehearsal) was significant $\left(r^{2}=.192, p<.001\right)$. A low beta weight in the regression analysis confirmed that retention interval is not a salient predictor of accuracy. In fact, importance rating was the only significant predictor, indicating that the common variance of distinctiveness, rehearsal, and importance substantially overlapped.

For the male participant there was a significant negative relation between retention interval and dating accuracy $(r=-.158, p<.004)$. There were also significant positive correlations for distinctiveness $(r=.157, p<$ $.004)$, rehearsal $(r=.358, p<.01)$, and importance $(r=$ $.334, p<.001)$. Again the regression analysis was significant $\left(r^{2}=.179, p<.01\right)$. In this case, however, retention interval and importance were significant.

For the female participant, $46 \%$ of the events were dated within +1 year of their occurrence; the figure was $40 \%$ for the male participant. The greater accuracy of the female was further reflected in the finding that $18.8 \%$ of her dates were within 1 week of the actual date, and $26.6 \%$ were within 4 weeks, whereas the male had only $9 \%$ within 1 week and $17.6 \%$ within 4 weeks.

\section{Integrative Analysis}

The model presented in Figure 1 served as the basis of integrative analysis. Path models for both performance scores for what-first items and know-remember judgments for all cue orders were evaluated using LISREL 8.3
(Jöreskog \& Sörbom, 1993). The strength of the path analysis in this context is that it allows for the integration of data from performance, the subjective ratings, and the experimenter ratings of distinctiveness, because the unit of analysis is events rather than individuals. Not all events received the subjective ratings, however, because participants had no recollection of some events and thus could not rate rehearsal, importance, or the qualitative nature of the recall experience. Thus, the data set for this analysis was 536 items for the female participant and 427 items for the male participant.

The evaluation of the model was influenced to some degree by the nature of the memories included in the sample, which can be characterized to some degree by the subjective ratings of rehearsal and importance. With regard to rehearsal, descriptive statistics indicated that few items were frequently rehearsed. Both participants anecdotally reported that they did not believe themselves to have discussed or thought about very many events other than those considered extremely significant. Consequently, the majority of items were rated at the low end of both scales. For rehearsal-talk, participants rated only $7.5 \%$ (female) and $17.1 \%$ (male) of the events at the upper end of the scale (ratings of 5, 6, or 7). The frequencies were similar for rehearsal-think. The two variables were correlated, .804 for the female participant and .796 for the male participant. This suggests either that memories that are frequently mentally rehearsed are also verbally rehearsed or that the participants had difficulty making this distinction. For importance, neither participant rated many events on the high end of the scale. Only $8 \%$ (female) and $7 \%$ (male) of the items received ratings 
Table 3

Path Coefficients From Maximum Likelihood Structural Analysis of Path Model for the Two Outcome Variables: Performance and Know-Remember Ratings

\begin{tabular}{|c|c|c|c|c|}
\hline \multirow[b]{2}{*}{ Path } & \multicolumn{2}{|c|}{ Performance Analyses $^{a}$} & \multicolumn{2}{|c|}{ Know-Remember Analyses } \\
\hline & Female & Male & Female & Male \\
\hline Rehearsal/outcome & $.44 *$ & $.24 *$ & $.79 *$ & $.76^{*}$ \\
\hline Log(time)/outcome & $-.93 *$ & $-.68 *$ & $-1.00 *$ & $-.36 *$ \\
\hline Log(time)/rehearsal & -.05 & $-.23 *$ & $.07 *$ & $.73 *$ \\
\hline Importance/outcome & $.25 *$ & $.31 *$ & $.07 *$ & $.12 *$ \\
\hline Importance/rehearsal & $.32 *$ & $.75 *$ & $.34 *$ & $.68 *$ \\
\hline Distinctiveness/outcome & .04 & .19 & $.38 *$ & .10 \\
\hline Distinctiveness/rehearsal & $.26^{*}$ & .12 & $.41 *$ & .27 \\
\hline$R^{2}$ (outcome) & $.28 *$ & $.27 *$ & $.61 *$ & $.61 *$ \\
\hline$N$ & 181 & 142 & 536 & 427 \\
\hline
\end{tabular}

a Outcome: Performance score data for what-first cue orders. $\quad$ boutcome: Remember-know ratings for all cue orders. ${ }^{*} p<.05$.

at the upper end of the importance scale (values of 5, 6 , 7 ). Whether this is idiosyncratic to these 2 individuals, a function of the many mundane events sampled, or a general trend among older adults is an empirical issue.

The summary results for the path analyses are presented in Table 3. The models were clearly good fits for both participants' performance data. For all four models tested there were $2 d f$, and the root mean square adjusted was 0.0 . Thus, the proposed model should not be rejected, but some aspects of the model fits merit our attention. The female participant's data conformed to the model both in the overall sense and in specific pathways, with some exceptions. For performance data, the effects associated with distinctiveness did not reach statistical significance. This finding partially reflects the paucity of highly distinctive events in the data set. For the male participant, rehearsal was less predictive of performance, but the skewed nature of the data distribution may be responsible. For both participants, the $r^{2}$ values for the Know-Remember Scale are much higher than those seen for the performance data, leading to the higher total effects for the rating variables. Also, the participants differed in the effects of importance and distinctiveness. These differences are discussed later in our evaluation of the model.

The model was evaluated for the entire 20 -year period. The relative contribution of the model variables may change over time. In particular, because the relation between retention interval and both performance and knowremember is curvilinear, retention interval would not account for differences in performance for events that took place within the interval between roughly 11 and 20 years ago. For example, analyzing the raw data, the simple correlation between $\log$ (time) and $\log$ (performance) for female performance is $-.246(p<.01)$ for Years $1-10$ and $.050(p>.10)$ for Years 11-20. This indicates a difference in the influence of time on memory. We discuss this issue below.

\section{DISCUSSION}

To summarize the primary results, the 2 participants in the study recalled information from autobiographical events at the rate of $40 \%$ (female) and $34 \%$ (male). Pretesting suggested that the differences in their performance were not due to $\operatorname{cog}$ nitive factors. Rather, the differences between the participants may be the result of a recorder effect, reflecting heightened self-relevance of the items for the female. The female selected all of the events, and though she included reference to her spouse, she had no consistent intention to do so. Thompson et al. (1996) found that recorders were more effective than nonrecorders at recognizing self-relevant events when they simply recorded events without any instruction to include activities involving their roommates. Given that our recorder received no instructions, it seems likely that the female participant recorded many events with high self-relevance.

Cue order was the most influential factor in memory performance. Initial what cues were more efficient than either who or where cues; and what-when was the most efficient combination. Retention curves for aggregated data did not show a systematic effect of time on memory performance, except for events initially prompted with what cues. Event distinctiveness was associated with better memory performance for both participants, even though there were relatively few highly distinctive events. With regard to the phenomenological experience of recalling events, time and rehearsal had the strongest relation to Know-Remember Scale ratings for both participants. Event importance contributed little unique variance to the ratings. Event distinctiveness was associated with the Know-Remember Scale for the female participant but not for the male participant. Across participants, the accuracy of memory dating was primarily a function of rated importance. Retention interval played a modest role for the male and a very weak role for the female. Below we discuss the impact of several variables in isolation and then integrate several in our discussion of path analysis.

\section{Cue Order and Cue Efficiency}

Cue order proved to be the most influential factor in memory retrieval. Cue-order effects overshadowed the effect of time and accounted for $18.4 \%$ (female) and 
$10.3 \%$ (male) of the variance in cued-recall scores. As hypothesized, the results replicated Wagenaar (1986, 1988) in that the initial what cues were more efficient than either who or where cues, and what-when was the most effective cue combination. However, unlike Wagenaar, we did not find where cues to be significantly better than who cues. In fact, who and where were found to be equal in their effectiveness, and neither reached the level of effectiveness reported by Wagenaar.

One likely explanation for these findings is that both who and where cues were low in distinctiveness for the present sample of events. Wagenaar (1986) suggested that cue distinctiveness is related to cue specificity. $\mathrm{He}$ only recorded events that were mutually exclusive and contained distinctive elements. For several reasons, this was less true in the present study. The information was not recorded systematically, with no intent to only record mutually exclusive events. The log contained relatively few completely distinctive events. Wagenaar traveled extensively at the time of his study, while our participants had settled into a daily routine of retirement that included a small group of friends and family with whom they engaged in most activities. Who often referred to only the 2 participants. In studies using younger participants, the range of daily events may be broader, offering more opportunities for distinctive experiences. Earlier in life, people may be active in a wider variety of activities related to their work, leisure, family life, their spouse's co-workers and friends, community, and neighborhood groups. For our participants, where was recorded as a city, a restaurant, or a friend's home; many sites appeared in the log several times per year. If the who and where elements are not diverse, they will be poor memory cues.

In contrast, what elements are by nature more distinctive and represent the core of the memory. In this study, what cues included such activities as 80th birthday parties or funerals, cues that were by definition unique, and consequently more effective as memory prompts. Thus, the prompt "funeral for Jean Smith" is more effective than "Mouser's funeral parlor." The pattern of results obtained here suggests that in most contexts future investigators may well wish to focus their efforts more selectively on what cues.

\section{Event Distinctiveness}

As in White (1982) and Brewer (1988), item selection in the present study purposefully included a range of memorable to mundane events. In our view, this provides a more valid estimate of memory accuracy. In fact, claims for the importance of distinctiveness can be evaluated only against the backdrop of memory for less distinctive events. The total number of completely unique events was only 22 for the female participant and 20 for the male participant. The inclusion of many events with low event distinctiveness contributes to the overall low retention rates observed. The participants were actually quite active, frequently golfing, dining out, and traveling to see family and friends. But the activities were repeti- tive. If their activity pattern is typical, it is likely that other older adults would show similar patterns of performance. In such a context, however, the age of the individual might be irrelevant, and all individuals might struggle to perform well.

For performance scores, the effect of distinctiveness for both participants was modest but linear, suggesting that changes in distinctiveness produce changes in cuedrecall performance. Thompson (1982) found a similar correlation for recall. The observed pattern for the female was also consistent with the results of Thompson et al. (1996) and Conway et al. (1996), whose studies spanned much shorter retention intervals for much younger participants. Those authors used cued recognition procedures that closely resembled the present Know-Remember Scale. The only exception to the general pattern regarding distinctiveness was for the male. We note that there was a similar pattern for importance ratings, which consistently predicted memory outcome for the female but not the male. These findings may reflect somewhat lower variance in his ratings but may also have something to do with the higher self-relevance of the memories for the female.

\section{Retention Interval}

Memory for events cued by initial what cues was the only subset of these data that resembled previous retention data. The curvilinear regression analyses suggested that for these data, it is not possible to distinguish between power and log models, but, as noted earlier, both of these models were superior to a linear model.

The retention curves showed an initially steep slope for the most recent intervals followed by a more gradual decline over the earlier years. Although it is important to demonstrate that the regularity of the data conforms to established models, the retention curves for what-cued events also showed some marked departures from the models for both participants. There were elevations in memory performance, as well as remember-know ratings, for both participants. As seen in Figures 4 and 5, however, these were not always for the same years. The elevated periods in the performance scores and the remember-know data bear some resemblance to the finding of "bumps" observed in cue-word studies (Fitzgerald, 1996). Two interpretations may be considered. The first, sampling error, is unlikely, given the adherence to randomization in event selection. Alternatively, one might propose a link to more effective encoding as a function of events from the affected intervals. The affected areas of the function that were consistent within and between participants represent events from Years 6-7 and 16-17. If cognitive factors were responsible, one might have expected adjoining years to be similarly affected, and this was not the case. Qualitative analysis support the alternative explanation for the elevated levels of recall. Content analysis of the log revealed a variety of landmark events, some shared by both participants and others individually experienced. For example, increased memory performance occurred around the time of the male par- 
ticipant's retirement, the couple's move to a new home in a distant state, the deaths of their mothers, their daughter's wedding, the birth of their first grandchild, and at times of serious health problems.

Anecdotally, participants described these "landmark" events consistent with definitions by Linton $(1975,1978)$, Neisser (1982), Robinson (1986), and Thompson (1982). They also used these events as a strategy in recall. For example, they remarked that these events provided a "time break," and they judged other events relative to these defining events. Linton $(1975,1978)$ pointed out the importance of "first-time" events, "one-of-a-kind" events, and "turning points" (Linton, 1982) in providing a reference for remembering and dating other events. The present data suggest that in addition to the normative bumps found in group data, idiosyncratic bumps might be found in individual data if a sufficient number of memories are sampled (Rubin, 1982).

\section{Retention and Inference}

The present study revealed examples of what Linton (1978) termed failure to discriminate between events and Brewer (1988) termed retrieval errors, in which individuals confuse one event with another. In the present study, this was typically the result of nondistinctive cues on the first or second page of the booklet. Participants often changed their answers as they received new information on subsequent pages. This occurred for low-distinctiveness events that permitted inferences based on semantic knowledge, also called general event knowledge (Conway, 1996), or repisodes (Neisser, 1982). Failure to discriminate was not limited, however, to mundane events and sometimes occurred for events considered rather distinctive, appearing only twice in the $\log$ (e.g., watching the United States lose the Ryder Cup). Participants had to guess which one. On the other hand, semantic information such as the time of year and the nature of the competition would be very helpful in reconstructing memories for each event.

Linton (1982) noted that repetition of events strengthened semantic memory, but had the opposite effect on episodic memory, making events less distinctive. A similar theme appears in the literature on false memories. In the Deese-Roediger/McDermott paradigm, participants are shown or hear lists of stimuli (needle, nurse) related to a target word (doctor) that is never actually presented. Participants frequently claim to have seen or heard the target word. Schacter has explained the false recognition of stimuli in the Deese-Roediger/McDermott paradigm in terms of a heavy reliance on semantic memory in the face of a lack of distinct perceptual cues (Schacter, Koutstaal, \& Norman, 1999). Thus, the impact of semantic similarity among events/items will depend on the nature and size of the item pool and the nature of the memory task.

\section{Integrative Analyses}

The integrative model presented in Figure 1 was examined independently for the female and male partici- pants for both performance scores and know-remember ratings. The strength of multiple evaluations of the model may be limited by the sample of memories that was skewed for importance, rehearsal, and distinctiveness. Of course, it seems likely that the underlying distribution of these characteristics for events in the lives of the participants, and perhaps many adults, is also skewed. All of the existing data sets in this domain comment on similar issues, suggesting that we are dealing with more than sampling issues. In fact, it is interesting to consider what factors might influence the reported frequencies of highly distinctive or important events. Much of what happens to us is not objectively very important, very distinctive, or very rehearsed. Even for events that seem important at the time, importance may fade quickly. Whether base rates vary with age, personality, gender, or social class is an empirical issue. In order to properly understand the relation among these variables and memory, some form of selective sampling will be necessary using data sets collected over extended periods. Thus, the outcomes reported in Table 3 are encouraging.

The model in Figure 1 strictly deals with assessments made after the time of encoding by either the participants or the experimenter. This distinguishes the present analysis from that of Conway et al. (1996), since their analyses included ratings made at both encoding and retrieval. Other differences are the inclusion of rehearsal and the use of recall rather than recognition measures, making direct comparisons problematic. The outcome of the path analysis provides clear support for the overall model for both participants for both outcome measures. In fact, because the fit is so high, comparisons to a saturated model (all possible paths specified) are rather pointless.

In the proposed model, rehearsal is seen as playing a key role. At least some of the influence of every other variable is carried through rehearsal. In part, this may reflect the implicit theories of the participants. For example, if he or she rates a memory as important, he/she may believe that it was talked about frequently; conversely, if he/she recalls talking about the memory frequently, this may be an indicator of event importance. The experimenter assigned distinctiveness ratings, however, suggesting that something other than the participants' beliefs is involved. Given that Conway et al. (1996) found significant relations between memory performance and participant ratings of self-relevance, affective valance, and intensity at encoding, the fact that we had no such ratings may be the major shortcoming of the present research. An evaluation of the magnitude of the direct and indirect influence of these variables over long intervals would be a useful contribution.

A shortcoming of models like that presented in Figure 1 is their nondynamic nature. As noted earlier, as the retention interval increases, the predictive value of time is reduced. In the present data set, for example, it would appear safe to say that recall probability is roughly equivalent whether an event took place 12 or 20 years ago. That 
probability depends upon whether the event is rehearsed, which reflects whether it is important, distinctive, or associated with a benchmark event. The shift from time dependent to time independent may be significant for developing an overall understanding of the influence of time on memory.

In conclusion, this study represents both the excitement and the frustrations of the study of autobiographicalmemory. By extending the diary study methodology to very long retention intervals, it reinforces the validity of conclusions that have been drawn on the basis of studies employing shorter intervals, the recall of materials such as yearbook photographs, and cue word methods. By employing multivariate methodologies, it has expanded our understanding of the interplay of variables such as rehearsal and importance. At the same time, it illustrates the frustrations that arise when the events available for study or the information recorded at encoding do not fit with idealized experimental design. Ultimately, however, we feel that the underlying regularities in autobiographical memory are sufficiently strong to support continuing naturalistic studies of the role that memory plays in everyday life.

\section{REFERENCES}

BAHRICK, H. P. (1984). Semantic memory content in permastore: Fifty years of memory for Spanish learned in school. Journal of Experimental Psychology: General, 113, 1-29.

BAHRICK, H. P., BAHRICK, P. O., \& WitTlinger, R. P. (1975). Fifty years of memory for names and faces: A cross-sectional approach. Journal of Experimental Psychology: General, 104, 54-75.

BARCLAY, C. R., \& Wellman, H. M. (1986). Accuracies and inaccuracies in autobiog raphical memories. Journal of Memory \& Language, 25, 93-103.

BeRnSTEIn, D. (1996). Involuntary autobiographical memory. Applied Cognitive Psychology, 10, 435-454.

BREWER, F. W. (1988). Memory for randomly sampled autobiographical events. In U. Neisser \& E. Winograd (Eds.), Remembering reconsidered: Ecological and traditional approaches to the study of memory (pp. 21-90). Cambridge: Cambridge University Press.

Cohen, G., \& FAUlKner, D. (1988). Life span changes in autobiographical memory. In M. M. Gruneberg, P. E. Morris, \& R. N. Sykes (Eds.), Practical aspects of memory (pp. 277-288). New York: Wiley.

CONWAY, M. A. (1992). A structural model of autobiographical memory. In M. A. Conway, D. C. Rubin, H. Spinler, \& W. A. Wagenaar (Eds.), Theoretical perspectives on autobiographical memory (pp. 167-194). Dordrecht: Kluwer.

CONWAY, M. A. (1995). Flashbulb memories. Hillsdale, NJ: Erlbaum.

Conway, M. A. (1996). Autobiographical knowledge and autobiographical memories. In D. C. Rubin (Ed.), Remembering our past: Studies in autobiographicalmemory (pp. 67-93). Cambridge: Cambridge University Press.

Conway, M. A., Collins, A. F., Gathercole, S. E., \& Anderson, S. J. (1996). Recollections of true and false autobiographical memories. Journal of Experimental Psychology: General, 12, 69-95.

Crocker, L., \& AlginA, J. (1986). Introduction to classical and modern test theory. New York: Holt, Rinehart \& Winston.

Crovitz, H. F., \& Schiffman, H. (1974). Frequency of episodic memories as a function of their age. Bulletin of the Psychonomic Society, 4, 517-518.

FitzGerald, J. M. (1986). Autobiographical memory: A developmental perspective. In D. C. Rubin (Ed.), Autobiographical memory (pp. 122-136). Cambridge: Cambridge University Press.

FITZGERALD, J. M. (1988). Vivid memories and the reminiscence phe- nomenon: The role of a self-narrative. Human Development, 31, 261273.

FitzGERALD, J. M. (1996). Intersecting meanings of reminiscence in adult development and aging. In D. C. Rubin (Ed.), Remembering our past: Studies in autobiographicalmemory (pp. 360-383). Cambridge: Cambridge University Press.

FitzGerAlD, J. M. (1999). Autobiographical memory and social cognition: Development of the remembered self in adulthood. In T. M. Hess \& F. Blanchard-Fields (Eds.), Social cognition and aging (pp. 145-171). New York: Academic Press.

FitzGerald, J. M., \& LAWRENCE, R. (1984). Autobiographical memory across the life-span. Journal of Gerontology, 39, 692-698.

GALTON, F. (1879). Psychometric experiments. Brain, 2, 149-162.

GALTON, F. (1883). Inquiries into human faculty and its development. London: Macmillan.

Hyman, I. E., Jr., Gilstrap, L. L., Decker, K., \& Wilkinson, C. (1998). Manipulating remember and know judgments of autobiographical memories: An investigation of false memory creation. Applied Cognitive Psychology, 12, 371-386.

JÖRESKOG, K., \& SÖRBOM, D. (1993). LISREL VIII users reference guide. Mooresville, IN: Scientific Software.

LARSEN, S. F. (1988). Remembering reported events: Memory for news in ecological perspective. In M. M. Gruneberg, P. E. Morris, \& R. N. Sykes (Eds.), Practical aspects of memory: Current research and issues (pp. 441-445). New York: Wiley.

Linton, M. (1975). Memory for real-world events. In D. A. Norman \& D. E. Rumelhart (Eds.), Explorations in cognition (pp. 376-404). San Francisco: Freeman.

LinTON, M. (1978). Real-world memory after 6 years: An in vivo study of very long-term memory. In M. M. Gruneberg, P. E. Morris, \& R. N. Sykes (Eds.), Practical aspects of memory (pp. 69-76). New York: Academic Press.

Linton, M. (1982). Transformations of memory in everyday life. In U. Neisser (Ed.), Memory observed: Remembering in natural contexts (pp. 77-81). San Francisco: Freeman.

NeISSE R, U. (1982). John Dean's memory: A case study. In U. Neisser (Ed.), Memory observed: Remembering in natural contexts (pp. 139159). San Francisco: Freeman.

RAJARAM, S. (1993). Remembering and knowing: Two means of access to the personal past. Memory \& Cognition, 21, 89-102.

Robinson, J. A. (1986). Temporal reference systems and autobiographical memory. In D. C. Rubin (Ed.), Autobiographicalmemory (pp. 159-188). Cambridge: Cambridge University Press.

RoEDIGER, H. L., III, \& CROWDER, R. G. (1982). A serial position effect in recall of United States presidents. In U. Neisser (Ed.), Memory observed: Remembering in natural contexts (pp. 230-238). San Francisco: Freeman.

RUBIN, D. C. (1982). On the retention function for autobiographical memory. Journal of Verbal Learning \& Verbal Behavior, 21, 21-38.

Rubin, D. C., \& KozIn, M. (1984). Vivid memories. Cognition, 16, 8195.

Rubin, D. C., \& Wenzel, A. E. (1996). One hundred years of forgetting: A quantitative description of retention. Psychological Review, 103, 734-760.

Rubin, D. C., Wetzler, S. E., \& Nebes, R. D. (1986). Autobiographical memory across the lifespan. In D. C. Rubin (Ed.), Autobiographical memory (pp. 202-221). Cambridge: Cambridge University Press.

SAlAmAN, E. (1970). A collection of moments: A study of involuntary memories. London: Longman.

Schacter, D. L., Koutstaal, W., \& Norman, K. A. (1999). False memories and aging. Trends in Cognitive Sciences, 1, 229-236.

SQuire, L., \& SLATER, P. C. (1982). An improved questionnaire technique. In U. Neisser (Ed.), Memory observed: Remembering in natural contexts (pp. 221-229). San Francisco: Freeman.

Thompson, C. P. (1982). Memory for unique personal events: The roommate study. Memory \& Cognition, 10, 324-332.

Thompson, C. P., Skrowronski, J. J., LARSEn, S. F., \& Betz, A. L. (1996). Autobiographical memory: Remembering what and remembering when. Mahwah, NJ: Erlbaum. 
Tulving, E. (1983). Elements of episodic memory. Oxford: Oxford University Press.

WAGENAAR, W. A. (1986). My memory: A study of autobiographical memory over 6 years. Cognitive Psychology, 18, 225-252.

WagenaAr, W. A. (1988). People and places in my memory: A study on cue specificity and retrieval from autobiographical memory. In M. M. Gruneberg, P. E. Morris, \& R. N. Sykes (Eds.), Practical aspects of memory: Current research and issues (pp. 228-233). New York: Wiley.

WetZler, S. E., \& Sweeney, J. A. (1986). Childhoodamnesia: An em- pirical demonstration. In D. C. Rubin (Ed.), Autobiographicalmemory (pp. 191-200). Cambridge: Cambridge University Press.

WheEler, M. A., Stuss, D. T., \& Tulving, E. (1997). Toward a theory of episodic memory: The frontal lobes and autonoetic consciousness. Psychological Bulletin, 121, 331-354.

White, R. T. (1982). Memory for personal events. Human Learning, $\mathbf{1}$, 171-183.

(Manuscript received February 4, 2003;

revision accepted for publication September 4, 2003.) 